\title{
Translational Research Program
}

National Cancer Institute

\section{Source}

National Cancer Institute. Translational Research Program. NCI Thesaurus. Code C82666.

A program within the National Cancer Institute designed to integ rate scientific advancements in the understanding of the biology of human cancer, with the development of new interventions for the prevention, diagnosis, and treatment of cancer, or populations at risk for cancer. This is accomplished by fostering broad interdisciplinary investigations that focus on bring ing discoveries from the laboratory to the clinic, and coordinating resources of the National Cancer Institute with those of academia, industry, and non-profit org anizations/foundations. 